\title{
Network synchronizability analysis: the theory of subgraphs and complementary graphs *
}

\author{
Zhisheng Duan ${ }^{1}$, Chao Liu ${ }^{1}$, and Guanrong Chen ${ }^{1,2}$ \\ ${ }^{1}$ State Key Laboratory for Turbulence and Complex Systems, Department of Mechanics and \\ Aerospace Engineering, College of Engineering, Peking University, Beijing 100871, P. R. China \\ 2 Department of Electronic Engineering, City University of Hong Kong, Hong Kong \\ Email: duanzs@pku.edu.cn, Fax: (8610)62765037
}

\begin{abstract}
In this paper, subgraphs and complementary graphs are used to analyze the network synchronizability. Some sharp and attainable bounds are provided for the eigenratio of the network structural matrix, which characterizes the network synchronizability, especially when the network's corresponding graph has cycles, chains, bipartite graphs or product graphs as its subgraphs.
\end{abstract}

Keywords. Complex network, Subgraph, Complementary graph, Synchronizability.

\section{Introduction}

Synchronization of complex networks has attracted increasing attention from many scientists, for its important applications in various areas of physical and biological sciences [3, 4, 6, 9, 10, 24, 26]. Synchronization is a ubiquitous phenomenon especially in social and biological networks where, quite often, it is desirable, e.g., in the consensus of multi-agent activities, while in some other cases it is undesirable, e.g., in traffic congestion [6, 16, 18, 19, 22, 23, 25]. The connection structure of a network plays an important role in its synchronization. Of particular interest in this concern is how the synchronizability depends on various structural parameters of the network, such as average distance, clustering coefficient, coupling strength, degree distribution and weight distribution, among others. Some interesting results have been established for such important problems based on the notions of master stability function and synchronized region [3, 5, 11, 12, 13, 16, 21, 29]. Some relationships between synchronizability and structural parameters of complex networks have also been

${ }^{*}$ This work was supported by the National Science Foundation of China under grant 60674093 and the City University of Hong Kong under the Research Enhancement Scheme and SRG grant 7002134. 
reported, e.g., smaller average network distance does not necessarily mean better synchronizability [19], therefore the betweenness centrality was proposed as a good indicator for synchronizability [10], and two networks with the same degree sequence can have different synchronizabilities [27. Some optimizing rules were established for the network synchronizability [6]. Moreover, how the network statistical properties influence the spectrum of the structural matrix was analyzed and reported in [2]. And the effects of graph operations on the network synchronizability were studied in [1]. Last but not least, complementary graphs were used to analyze the network synchronizability in [7]. Motivated by all these research works, this paper attempts to further establish some sharp upper bounds for the network synchronizability based on the theory of subgraphs and complementary graphs.

Consider a dynamical network consisting of $N$ coupled identical nodes, with each node being an $n$-dimensional dynamical system, described by

$$
\dot{x}_{i}=f\left(x_{i}\right)-c \sum_{j=1}^{N} a_{i j} H\left(x_{j}\right), i=1,2, \cdots, N,
$$

where $x_{i}=\left(x_{i 1}, x_{i 2}, \cdots, x_{i n}\right) \in \mathbb{R}^{n}$ is the state vector of node $i, f(\cdot): \mathbb{R}^{n} \rightarrow \mathbb{R}^{n}$ is a smooth vector-valued function, constant $c>0$ represents the coupling strength, $H(\cdot): \mathbb{R}^{n} \rightarrow \mathbb{R}^{n}$ is called the inner linking function, and $A=\left(a_{i j}\right)_{N \times N}$ is called the outer coupling matrix or structural matrix, which represents the coupling configuration of the entire network. This paper only considers the case that the network is diffusively connected, i.e., $A$ is irreducible and its entries satisfy $a_{i i}=-\sum_{j=1, j \neq i}^{N} a_{i j}, i=1,2, \cdots, N$. Further, suppose that, if there is an edge between node $i$ and node $j$, then $a_{i j}=a_{j i}=-1$, i.e., $A$ is a Laplacian matrix corresponding to network (1)). Therefore, 0 is an eigenvalue of $A$ with multiplicity 1, and all the other eigenvalues of $A$ are strictly positive, which are denoted by

$$
0=\lambda_{1}<\lambda_{2} \leq \lambda_{3} \leq \cdots \leq \lambda_{N}=\lambda_{\max }
$$

The dynamical network (11) is said to achieve (asymptotical) synchronization if $x_{1}(t) \rightarrow x_{2}(t) \rightarrow$ $\cdots \rightarrow x_{N}(t) \rightarrow s(t)$, as $t \rightarrow \infty$, where, because of the diffusive coupling configuration, the synchronous state $s(t) \in \mathbb{R}^{n}$ is a solution of an individual node, i.e., $\dot{s}(t)=f(s(t))$.

It is well known [3] that the eigenratio $r(A)=\frac{\lambda_{2}}{\lambda_{N}}$ of network structural matrix $A$ characterizes the network synchronizability: the larger the $r(A)$, the better the synchronizability. In this paper, $r(A)$ will be used as a synchronizability index for the networks with structural matrix $A$. The relationships between $r(A)$ and network structural characteristics such as average distance, node betweenness, degree distribution, clustering coefficient, etc. have been studied [7, 2, 27, 28]. A well-known upper bound for the eigenratio $r(A)$ in graph theory is $r(A) \leq \frac{d_{\min }}{d_{\max }}$, where $d_{\min }$ and $d_{\text {max }}$ denote the smallest and largest degrees of the corresponding graph to the network [27]. To the best of our knowledge, besides this coarse bound, there are no sharp upper bounds available 
in the literature today. Some sharp upper bounds for $r(A)$ are derived in this paper by using graph-theoretical methods.

The rest of this paper is organized as follows. In Section 2, some necessary preliminaries in graph theory are provided. In Section 3, for a given graph $G$, some upper bounds are derived for the eigenratio $r(G)$ when $G$ has cycles as its subgraphs. In Section 4, some lower bounds for the largest eigenvalue of a graph are presented when $G$ has other subgraphs, such as chains, bipartite graphs and product graphs. The paper is concluded by the last section.

Throughout this paper, for any given undirected graph $G$, eigenvalues of $G$ mean eigenvalues of its corresponding Laplacian matrix. Notations for graphs and their corresponding Laplacian matrices are not differentiated, and networks and their corresponding graphs are not distinguished, unless otherwise indicated.

\section{Preliminaries}

For a given graph $G$, let $\mathcal{V}$ and $\mathcal{E}$ denote the sets of nodes and edges of $G$, respectively. A graph $G_{1}$ is called an induced subgraph of $G$, if the node set $\mathcal{V}_{1}$ of $G_{1}$ is a subset of $\mathcal{V}$ and the edges of $G_{1}$ are all edges among nodes $\mathcal{V}_{1}$ in $\mathcal{E}$. The complementary graph of $G$, denoted by $G^{c}$, is the graph containing all the nodes of $G$ and all the edges that are not in $G$. In this paper, subgraphs and complementary graphs are used to discuss the network synchronizability. For this purpose, the following lemmas are needed.

Lemma 1 [14, 15] For any given graph $G$ of size $N$, its nonzero eigenvalues indexed as in (2) grow monotonically with the number of added edges; that is, for any added edge $e, \lambda_{i}(G+e) \geq \lambda_{i}(G)$, $i=1, \cdots, N$.

Lemma 2 [14] For any given connected graph $G$ of size $N$, its largest eigenvalue $\lambda_{N}$ satisfies $\lambda_{N} \geq d_{\max }+1$, with equality if and only if $d_{\max }=N-1$, where $d_{\max }$ is the maximum degree of G.

Lemma 3 [8, 27] For any cycle $C_{N}$ at $N(\geq 4)$ nodes, its eigenvalues are given by $\mu_{1}, \cdots, \mu_{N}$ (not necessarily ordered) with $\mu_{1}=0$ and

$$
\mu_{k+1}=3-\frac{\sin \left(\frac{3 k \pi}{N}\right)}{\sin \left(\frac{k \pi}{N}\right)}, k=1, \cdots, N-1 .
$$

By Lemma 3, one knows that, for any cycle $C_{N}$ at $N(\geq 4)$ nodes, if $N$ is even, its largest eigenvalue is 4 ; if $N$ is odd, its largest eigenvalue is $\lambda_{N}\left(C_{N}\right)=3-\frac{\sin \left(\frac{3(N-1) \pi}{2 N}\right)}{\sin \left(\frac{(N-1) \pi}{2 N}\right)}$.

Obviously, for cycles with odd numbers of nodes, the largest eigenvalue converges to 4 as the number of its lengths tends to $+\infty$. For eigenvalues of graphs and complementary graphs, the following lemma is useful (see [14, 15] and references therein). 
Lemma 4 For any given graph $G$ of size $N$, the following statements hold:

(i) $\lambda_{N}(G)$, the largest eigenvalue of $G$, satisfies $\lambda_{N}(G) \leq N$.

(ii) $\lambda_{N}(G)=N$ if and only if $G^{c}$ is disconnected.

(iii) If $G^{c}$ is disconnected and has (exactly) $q$ connected components, then the multiplicity of $\lambda_{N}(G)=N$ is $q-1$.

(iv) $\lambda_{i}\left(G^{c}\right)+\lambda_{N-i+2}(G)=N, \quad 2 \leq i \leq N$.

For a given graph, generally its largest eigenvalue is easier to compute than the smallest nonzero eigenvalue. Hence, by Lemma 4, one can obtain the smallest nonzero eigenvalue by computing the largest eigenvalue of its complementary graph.

Corollary 1 For any given graph $G$ of size $N$, if its second smallest eigenvalue equals its smallest node degree, i.e., $\lambda_{2}(G)=d_{\min }(G)$, then $G$ or $G^{c}$ is disconnected; if $\lambda_{2}(G)>d_{\min }(G)$, then $G$ is a complete graph; if both $G$ and $G^{c}$ are connected, then $\lambda_{2}(G)<d_{\min }(G)$.

Proof If $G^{c}$ is connected, then $\lambda_{\max }\left(G^{c}\right)=N-\lambda_{2}(G)=N-d_{\min }\left(G_{1}\right)=d_{\max }\left(G^{c}\right)+1$. By Lemma $2, d_{\max }\left(G^{c}\right)=N-1$, so $G$ is disconnected. Further, if $\lambda_{2}(G)>d_{\min }(G)$, by Lemma 4, $\lambda_{N}\left(G^{c}\right)<d_{\max }+1$. Combining with Lemma $2, G^{c}$ can only have isolated nodes, i.e., $G$ is a complete graph. The third statement holds obviously.

Combining Lemmas 1 and 2 with Corollary 1, one can easily get the following result.

Corollary 2 For any given connected graph $G$ and every its induced subgraph $G_{1}$, one has $\lambda_{\max }(G) \geq \lambda_{\max }\left(G_{1}\right)$, so the synchronizability index of $G$ satisfies $r(G)<\frac{d_{\min }(G)}{\lambda_{\max }\left(G_{1}\right)}$; if both $G$ and $G^{c}$ are connected, then $r(G)<\frac{d_{\min }}{d_{\max }+1}$.

Since subgraphs have less nodes, this corollary is useful when a graph $G$ contains some canonical graphs whose largest eigenvalues can be easily obtained as subgraphs (see the section below for further discussion).

Corollary 3 For a given graph $G$, if the largest eigenvalue of $G^{c}$ is $\lambda_{\max }=d_{\max }\left(G^{c}\right)+\alpha$, then $\lambda_{2}(G)=d_{\min }(G)+1-\alpha$. Consequently, the synchronizability index of $G$ satisfies $r(G)=$ $\frac{d_{\min }(G)+1-\alpha}{\lambda_{\max }(G)} \leq \frac{d_{\min }(G)+1-\alpha}{d_{\max }(G)+1}$.

By Lemma 2, generally $\alpha \geq 1$, so the bound in Corollary 3 is better than the one in Corollary 2 .

\section{Graphs having cycles as subgraphs}

In this section, an even cycle means a cycle with an even number of nodes and an odd cycle means a cycle with an odd number of nodes. By the discussion in the above section, one can get the following results.

Lemma 5 For any even cycle, -2 is an eigenvalue of its adjacency matrix. For any odd cycle with $n_{1}$ nodes, $-1+\frac{\sin \left(\frac{3\left(n_{1}-1\right) \pi}{2 n_{1}}\right)}{\sin \left(\frac{\left(n_{1}-1\right) \pi}{2 n_{1}}\right)}$ is an eigenvalue of its adjacency matrix. 
Proof Lemma 3 directly leads to the result.

Theorem 1 For any given graph $G$, suppose $G_{1}$ is its induced subgraph composing of all nodes of $G$ with the maximum degree $d_{\max }(G)$, and $\mathcal{G}_{1}$ is the induced subgraph of $G^{c}$ composing of all nodes of $G^{c}$ with the maximum degree $d_{\max }\left(G^{c}\right)$. Then, the following statements hold:

(i) If both $G_{1}$ and $\mathcal{G}_{1}$ have even cycles as induced subgraphs, then $\lambda_{\max }(G) \geq d_{\max }(G)+2$ and $\lambda_{\max }\left(G^{c}\right) \geq d_{\max }\left(G^{c}\right)+2$. Consequently, the synchronizability index of $G$ satisfies $r(G) \leq$ $\frac{d_{\min }(G)-1}{d_{\max }(G)+2}$.

(ii) If both $G_{1}$ and $\mathcal{G}_{1}$ have odd cycles as induced subgraphs, and if the longest odd cycle of $G_{1}$ has $n_{1}$ nodes and the longest odd cycle of $\mathcal{G}_{1}$ has $n_{2}$ nodes, then $\lambda_{\max }(G) \geq d_{\max }(G)+1-\frac{\sin \left(\frac{3\left(n_{1}-1\right) \pi}{2 n_{1}}\right)}{\sin \left(\frac{\left(n_{1}-1\right) \pi}{2 n_{1}}\right)}$ and $\lambda_{\max }\left(G^{c}\right) \geq d_{\max }\left(G^{c}\right)+1-\frac{\sin \left(\frac{3\left(n_{2}-1\right) \pi}{2 n_{2}}\right)}{\sin \left(\frac{\left(n_{2}-1\right) \pi}{2 n_{2}}\right)}$. Consequently, the synchronizability index of $G$ satisfies $r(G) \leq \frac{d_{\min }(G)+\frac{\sin \left(\frac{3\left(n_{2}-1\right) \pi}{2 n_{2}}\right)}{\sin \left(\frac{\left(n_{2}-1\right) \pi}{2 n_{2}}\right)}}{d_{\max }(G)+1-\frac{\sin \left(\frac{3\left(n_{1}-1\right) \pi}{2 n_{1}}\right)}{\sin \left(\frac{\left(n_{1}-1\right) \pi}{2 n_{1}}\right)}}$.

(iii) If $G_{1}$ has an even cycle as an induced subgraph, and $\mathcal{G}_{1}$ has odd cycles as induced subgraphs with the longest odd cycle having $n_{2}$ nodes, then $\lambda_{\max }(G) \geq d_{\max }(G)+2$ and $\lambda_{\max }\left(G^{c}\right) \geq$ $d_{\text {max }}\left(G^{c}\right)+1-\frac{\sin \left(\frac{3\left(n_{2}-1\right) \pi}{2 n_{2}}\right)}{\sin \left(\frac{\left(n_{2}-1\right) \pi}{2 n_{2}}\right)}$. Consequently, the synchronizability index of $G$ satisfies $r(G) \leq \frac{d_{\min }(G)+\frac{\sin \left(\frac{3\left(n_{2}-1\right) \pi}{2 n_{2}}\right)}{\sin \left(\frac{\left(n_{2}-1\right) \pi}{2 n_{2}}\right)}}{d_{\max }(G)+2}$.

(iv) If $G_{1}$ has odd cycles as induced subgraphs with the longest odd cycle having $n_{1}$ nodes and $\mathcal{G}_{1}$ has an even cycle as an induced subgraph, then $\lambda_{\max }(G) \geq d_{\max }(G)+1-\frac{\sin \left(\frac{3\left(n_{1}-1\right) \pi}{2 n_{1}}\right)}{\sin \left(\frac{\left(n_{1}-1\right) \pi}{2 n_{1}}\right)}$ and $\lambda_{\max }\left(G^{c}\right) \geq d_{\max }\left(G^{c}\right)+2$. Consequently, the synchronizability index of $G$ satisfies

$r(G) \leq \frac{d_{\min }(G)-1}{d_{\max }(G)+1-\frac{\sin \left(\frac{3\left(n_{1}-1\right) \pi}{2 n_{1}}\right)}{\sin \left(\frac{\left(n_{1}-1\right) \pi}{2 n_{1}}\right)}}$.

Proof (i) By Lemmas 3 and 5, for any even cycle, its largest eigenvalue is 4 and -2 is an eigenvalue of its adjacency matrix. Let $L_{1}$ be the sub-matrix of the Laplacian matrix of $G$ related to all the nodes in $G_{1}$, and $A\left(G_{1}\right)$ be the adjacency matrix of $G_{1}$. Then, $A\left(G_{1}\right)$ contains a submatrix which is the adjacency matrix of the corresponding even cycle. Therefore, one has

$$
\left(d_{\max }(G)+2\right) I-L_{1}=2 I+A\left(G_{1}\right) \ngtr 0 .
$$

In fact, the sub-matrix of $2 I+A\left(G_{1}\right)$ corresponding to the even cycle has a zero eigenvalue. This implies that the largest eigenvalue of $G_{1}$ is larger than or equal to $d_{\max }+2$. Then, by Lemma 1 , $\lambda_{\max }(G) \geq d_{\max }+2$. Similarly, $\lambda_{\max }\left(G^{c}\right) \geq d_{\max }+2$.

Further, suppose the number of nodes of $G$ is $N$. Then, the smallest nonzero eigenvalue of $G$ is $\lambda_{2}=N-\lambda_{\max }\left(G^{c}\right)$ and the minimum node degree of $G$ is $d_{\min }(G)=N-1-d_{\max }\left(G^{c}\right)$. If $\lambda_{\max }\left(G^{c}\right) \geq d_{\max }\left(G^{c}\right)+2$, then $\lambda_{2} \leq N-d_{\max }\left(G^{c}\right)-2=d_{\min }(G)-1$. Therefore, Theorem 1 (i) obviously holds. 
(ii) Follows easily from (i) and Lemma 5.

(iii) and (iv) can be similarly proved.

Remark 1 For a given graph $G$, clearly, the bounds given in Theorem 1 for the synchronizability index $r(G)$ are better than the general bound $r(G) \leq \frac{d_{\min }}{d_{\max }}$. And the existence of cycles can be easily tested by drawing graphs. Since the networks with good synchronizabilities always have homogeneous degree distributions [6, 10, Theorem 1 is especially useful for estimating the synchronizabilities of homogeneous networks.

Remark 2 The smallest even cycle is cycle $C_{4}$, and its complementary graph $C_{4}^{c}$ has two separated edges. $C_{4}$ and $C_{4}^{c}$ are very important in graph theory [15]. A graph has a $C_{4}$ as an induced subgraph if and only if $G^{c}$ has $C_{4}^{c}$ as an induced subgraph.

Remark 3 The cycle $C_{3}$ is the smallest cycle. If a graph $G$ contains only $C_{3}$ cycles, its largest eigenvalue satisfies $\lambda_{\max }(G) \geq d_{\max }+1$.

Example 1 Consider cycle $C_{5}$ in Fig. 1. Its complementary graph is also $C_{5}$ (see Fig. 2). Testing the eigenvalues of $C_{5}$ and its synchronizability, one finds that they attain the exact bounds in Theorem 1 (ii).

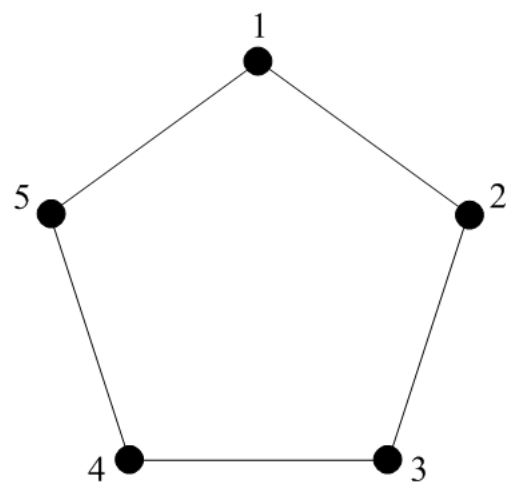

Fig. 1 Graph $C_{5}$
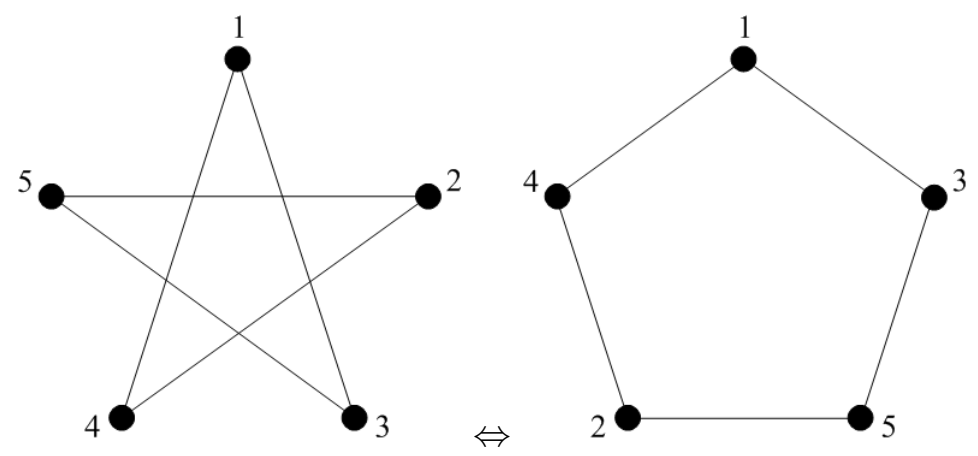

Fig. 2 Graph $C_{5}^{c}$

Example 2 Consider graph $\Gamma_{1}$ in Fig. 3. Its complementary graph is $C_{6}$ (see Fig. 4). Testing the eigenvalues of $\Gamma_{1}$ and its synchronizability, one finds that they attain the exact bounds in Theorem 1 (i).
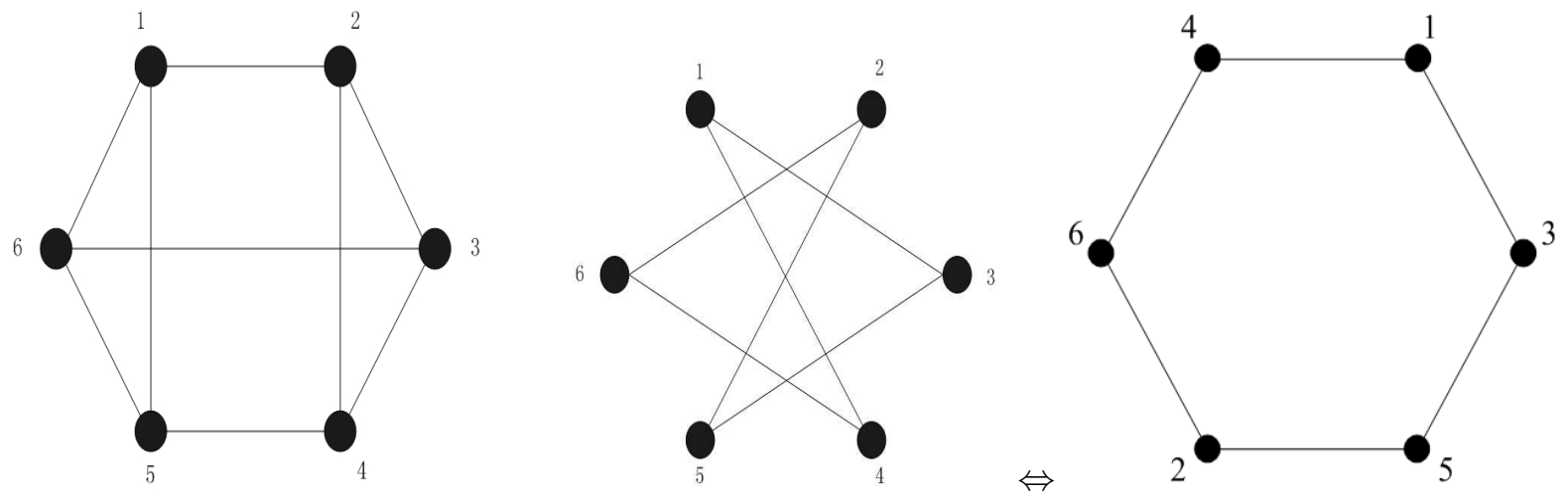


\section{Applications of other subgraphs}

According to the discussion in the above section, cycles are very important for estimating the network synchronizability. In this section, consider the estimation of the largest eigenvalue of a given graph by its subgraphs. First, the following lemma for adjacency matrices is needed.

Lemma 6 [17] Given a graph $G$, the smallest eigenvalue of its adjacency matrix $A(G)$ satisfies $\lambda_{\min }(A(G)) \leq d_{\max }(G)-\lambda_{\max }(G)$.

By this lemma, one can get the following result.

Theorem 2 For a given graph $G$, let $H$ be a subgraph of $G$ containing all nodes of $G$ with the same node degree $d$. Let $H_{1}$ be a subgraph of $H$. Then, the largest eigenvalue of $G$ satisfies $\lambda_{\max }(G) \geq d+\lambda_{\max }\left(H_{1}\right)-d_{\max }\left(H_{1}\right)$.

Proof It follows from Lemma 6 and the proof of Theorem 1.

Remark 4 In the above section, cycles as subgraphs have the same node degrees. So the smallest eigenvalues of the adjacency matrices of cycles can be exactly computed. In Theorem 2, for a general subgraph $H_{1}$, its node degrees are not necessarily the same. In this case, Lemma 6 is very useful for estimating the largest eigenvalues.

In what follows, consider some canonical subgraphs which can be used to estimate the largest eigenvalues of the original graphs. All the results can be combined with the results in the above section to obtain upper bounds for the network synchronizability.

\subsection{Graphs having chains as subgraphs}

A chain is a nearest-neighbor coupled graph without an edge between the first and the last nodes. For a given chain $P_{N}$ with $N$ nodes, it is well known that its largest and smallest nonzero eigenvalues are $2\left(1+\cos \left(\frac{\pi}{N}\right)\right)$ and $2\left(1-\cos \left(\frac{\pi}{N}\right)\right)$, respectively [8, 14, 27]. Obviously, the largest eigenvalue of a chain converges to 4 as its length tends to $+\infty$. According to the above discussion, one can establish the following result.

Theorem 3 For a given graph $G$, let $G_{1}$ be a subgraph of $G$ containing all nodes of $G$ with the maximum degree. Suppose $G_{1}$ contains a chain $P_{k}$ with $k$ nodes as its subgraph. Then, the largest eigenvalue of $G$ satisfies $\lambda_{\max }(G) \geq d_{\max }+2 \cos \left(\frac{\pi}{N}\right)$.

Proof Theorems 1 and 2 directly lead to the conclusion.

Note that chain $P_{4}$ is a very important graph in graph theory [15]. The complementary graph of $P_{4}$ is still a $P_{4}$ graph. Therefore, a graph $G$ has $P_{4}$ as a subgraph if and only if $G^{c}$ has $P_{4}$ as a subgraph. 


\subsection{Graphs having bipartite graphs as subgraphs}

Let $G_{1}\left(\mathcal{V}_{1}, \mathcal{E}_{1}\right)$ and $G_{2}\left(\mathcal{V}_{2}, \mathcal{E}_{2}\right)$ be two graphs on disjoint sets of $n_{1}$ and $n_{2}$ nodes, respectively, where $\mathcal{V}_{i}$ and $\mathcal{E}_{i}$ are the corresponding sets of nodes and edges. Their disjoint union $G_{1}+G_{2}$ is the graph $G_{1}+G_{2}=\left(\mathcal{V}_{1} \cup \mathcal{V}_{2}, \mathcal{E}_{1} \cup \mathcal{E}_{2}\right)$. Then, a bipartite graph $G_{1} * G_{2}$ generated by $G_{1}$ and $G_{2}$ is a new graph composing of $G_{1}+G_{2}$ and the new edges connecting each node of $G_{1}$ to every node of $G_{2}$, as detailed in [1, 15, 20]. It is well known that the largest eigenvalue of the bipartite graph $G_{1} * G_{2}$ is $\lambda_{\max }\left(G_{1} * G_{2}\right)=n_{1}+n_{2}$, i.e., the sum of the numbers of nodes of $G_{1}$ and $G_{2}$. In fact, the complementary graphs of bipartite graphs are always disconnected.

By Lemma 6, for a bipartite graph $G_{1} * G_{2}$, as discussed above, one can has $\lambda_{\min }\left(A\left(G_{1} * G_{2}\right)\right) \leq$ $d_{\max }\left(G_{1} * G_{2}\right)-n_{1}-n_{2}$.

Theorem 4 For a given graph $G$, let $H$ be a subgraph of $G$ containing all nodes of $G$ with the same degree $d$. Suppose $H$ contains a bipartite subgraph $H_{1} * H_{2}$, and the numbers of nodes of $H_{1}$ and $H_{2}$ are $n_{1}$ and $n_{2}$, respectively. Then, the largest eigenvalue of $G$ satisfies $\lambda_{\max }(G) \geq$ $d+n_{1}+n_{2}-d_{\max }\left(H_{1} * H_{2}\right)$.

Proof This is a direct result of Theorem 2 for bipartite subgraphs.

Example 3 Consider graph $\Gamma_{2}$ in Fig. 5. Obviously, $\Gamma_{2}$ has a bipartite graph $H$ as its subgraph which is composed of all nodes with degree 6 from $\Gamma_{2}$. The largest eigenvalue of this bipartite graph is 8 . So, by Theorem $4, \lambda_{\max }\left(\Gamma_{2}\right) \geq 9$. On the other hand, the maximum degree of the complementary graph $\Gamma_{2}^{c}$ of $\Gamma_{2}$ is 8 . And the nodes with degree 8 in $\Gamma_{2}^{c}$ form a cycle $C_{4}$. By Theorem 1 , the smallest nonzero eigenvalue of $\Gamma_{2}$ satisfies $\lambda_{2}\left(\Gamma_{2}\right) \leq d_{\min }\left(\Gamma_{2}\right)-1=2$. So, $r\left(\Gamma_{2}\right) \leq \frac{2}{9}$. Simply computing the Laplacian eigenvalues of $\Gamma_{2}$, one has $\lambda_{2}=1.7251$ and $\lambda_{\max }=9.2749$. Consequently, $r\left(\Gamma_{2}\right)=\frac{1.7251}{9.2749} \approx 0.176$. Therefore, the theorems in this paper successfully give the upper integer of the largest eigenvalue and the lower integer of the smallest nonzero eigenvalue of $\Gamma_{2}$.

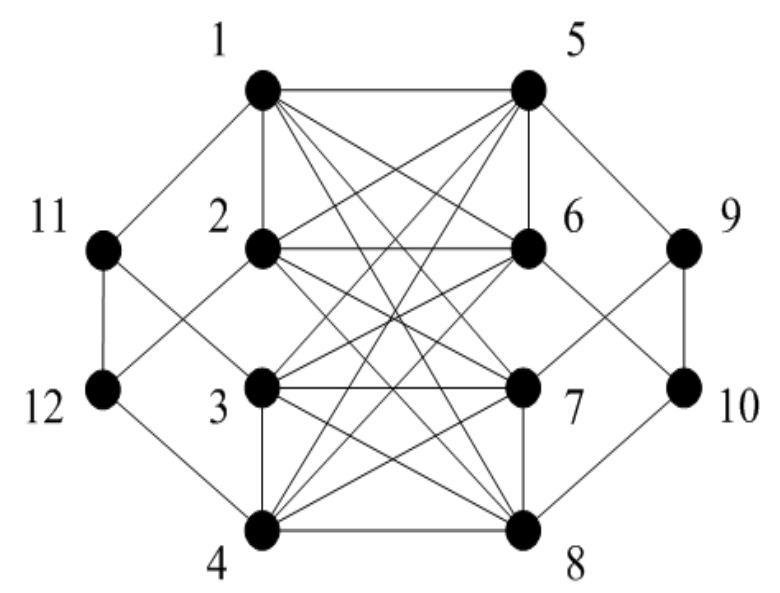

Fig. 5 Graph $\Gamma_{2}$ 


\subsection{Graphs having product graphs as subgraphs}

Let $G_{1}\left(\mathcal{V}_{1}, \mathcal{E}_{1}\right)$ and $G_{2}\left(\mathcal{V}_{2}, \mathcal{E}_{2}\right)$ be two nonempty graphs. Their product graph $G_{1} \times G_{2}$ is a graph with node set $\mathcal{V}_{1} \times \mathcal{V}_{2}$, and $\left(x_{1}, x_{2}\right)\left(y_{1}, y_{1}\right)$ is an edge in $\mathcal{E}\left(G_{1} \times G_{2}\right)$ if and only if either $x_{2}=y_{2}$ with $x_{1} y_{1} \in \mathcal{E}\left(G_{1}\right)$ or $x_{1}=y_{1}$ with $x_{2} y_{2} \in \mathcal{E}\left(G_{2}\right)$. One may view $G_{1} \times G_{2}$ as the graph obtained from $G_{1}$ by replacing each of its nodes with a copy of $G_{2}$ and each of its edges with the number of nodes of $G_{2}$ edges joining the corresponding nodes of $G_{2}$ in two copies, as detailed in [1, 15].

It is well known that the largest eigenvalue of the product graph $G_{1} \times G_{2}$ is $\lambda_{\max }\left(G_{1} \times G_{2}\right)=$ $\lambda_{\max }\left(G_{1}\right)+\lambda_{\max }\left(G_{2}\right)$.

Theorem 5 For a given graph $G$, let $H$ be a subgraph of $G$ containing all nodes of $G$ with the same node degree $d$. Suppose $H$ contains a product graph $H_{1} \times H_{2}$ as its subgraph. Then, the largest eigenvalue of $G$ satisfies $\lambda_{\max }(G) \geq d+\lambda_{\max }\left(H_{1}\right)+\lambda_{\max }\left(H_{2}\right)-d_{\max }\left(H_{1} \times H_{2}\right)$.

Example 4 Consider graph $\Gamma_{3}$ in Fig. 6. Obviously, all nodes of $\Gamma_{3}$ have degree 4 . And $\Gamma_{3}$ has a product graph $C_{4} \times P_{3}$ (nodes 1 to 12) as its subgraph, where $P_{3}$ denotes a chain with three nodes. The largest eigenvalue of this product subgraph is 7 . So, by Theorem 5 or Lemma $1, \lambda_{\max }\left(\Gamma_{3}\right) \geq 7$. On the other hand, the complementary graph $\Gamma_{3}^{c}$ of $\Gamma_{3}$ has a bipartite graph as its subgraph which is composed of nodes 1 to 4 and nodes 9 to 12 . By Theorem 4 , similarly to Example 3, the largest eigenvalue of $\Gamma_{3}^{c}$ satisfies $\lambda\left(\Gamma_{3}^{c}\right) \geq d_{\max }\left(\Gamma_{3}^{c}\right)+3$. Thus, by Corollary 3 , the smallest nonzero eigenvalue of $\Gamma_{3}$ satisfies $\lambda_{2}\left(\Gamma_{3}\right) \leq d_{\min }\left(\Gamma_{2}\right)-2=2$. So, $r\left(\Gamma_{3}\right) \leq \frac{2}{7}$. Simply computing the Laplacian eigenvalues of $\Gamma_{3}$, one has $\lambda_{2}=1.2679$ and $\lambda_{\max }=7.4142$. Consequently, $r\left(\Gamma_{2}\right)=\frac{1.2679}{7.4142} \approx 0.171$. Similarly to Example 3 , the corresponding theorems proved in this paper successfully give the upper integer of the largest eigenvalue and the lower integer of the smallest nonzero eigenvalue of $\Gamma_{3}$.

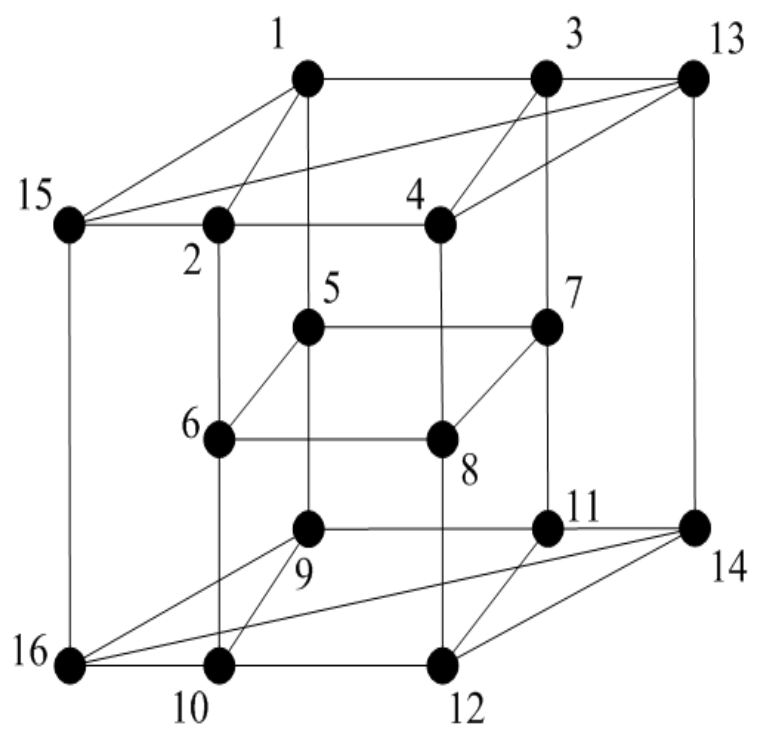

Fig. 6 Graph $\Gamma_{3}$ 


\subsection{The maximum disconnected subgraph}

Given a graph $G$ of size $N$, suppose $G_{1}$ is an induced subgraph of $G$ of size $n_{1}$, and $G_{1}$ is disconnected. $G_{1}$ is called a maximum disconnected subgraph, if the node number of any other disconnected subgraph of $G$ is less than or equal to $n_{1}$.

Theorem 5 For a given connected graph $G$ of size $N$, if the node number of its maximum disconnected subgraph is $n_{1}$, then the smallest nonzero eigenvalue of $G$ satisfies $\lambda_{2} \leq N-n_{1}$. Consequently, $r(G) \leq \frac{N-n_{1}}{d_{\max }(G)+1}$.

Proof Suppose $G_{1}$ is a maximum disconnected subgraph of size $n_{1}$. Then, by Lemma 4 , $\lambda_{\max }\left(G_{1}^{c}\right) \geq n_{1}$. Further, $\lambda_{\max }\left(G^{c}\right) \geq n_{1}$, so $\lambda_{2}(G) \leq N-n_{1}$.

Example 5 Consider graph $\Gamma_{4}$ in Fig. 7. By deleting node 3 or 6 , one can verify that the node number of its maximum disconnected subgraph is 7 . So, $\lambda_{2}\left(\Gamma_{4}\right) \leq 1$. Combining Lemma 2 and Theorem 6, one has $r\left(\Gamma_{4}\right)<\frac{1}{5}$. It is well known that graphs as in Fig. 7 have large node and edge betweenness centralities therefore have bad synchronizabilities. Theorem 6 , based on the theory of subgraphs and complementary graphs, gives an explanation why such graphs indeed have bad synchronizabilities.

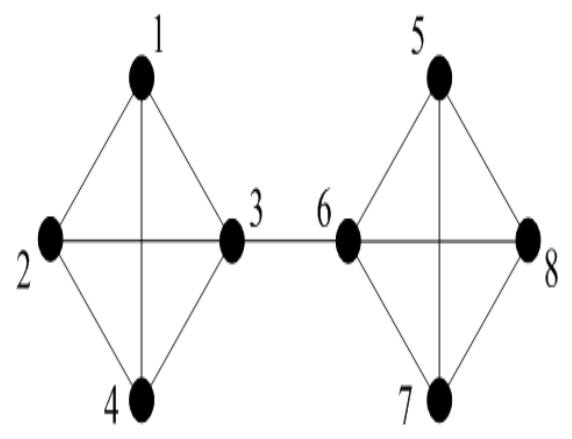

Fig. 7 Graph $\Gamma_{4}$

Remark 5 From Examples 3 to 5, it can be seen that actually one does not need to draw out the complementary graphs; the properties of complementary graphs can be analyzed using the original graphs.

\section{Conclusion}

In this paper, some relatively tight upper bounds have been obtained for the network synchronizability based on the theory of subgraphs and complementary graphs. Especially, some sharp bounds are given for the eigenratioes and the largest eigenvalues when a graph has some canonical subgraphs, such as cycles, chains, bipartite graphs and product graphs. Considering that the networks with good synchronizabilities typically have homogeneous degree distributions, the results obtained in this paper are particularly useful for estimating the synchronizabilities of homogeneous networks. 
And for a given network, its corresponding graph as well as its complementary graph and subgraphs can be easily obtained. This paper shows that better understanding and helpful manipulation of subgraphs and complementary graphs are very useful for enhancing the network synchronizability. Therefore, the graph-theoretical method provided in this paper is deemed important in the study of network synchronization problems.

\section{References}

[1] F. M. Atay, T. Bıyıkoğlu. Graph operations and synchronization of complex networks, Phys. Rev. E, 72: 016217, 2005.

[2] F. M. Atay, T. Biyıkoğlu, J. Jost. Network synchronization: Spectral versus statistical properties, Physica D, 224: 35-41, 2006.

[3] M. Barahona, L. M. Pecora. Synchronization in small-world systems, Phys. Rev. Lett., 89(5): 054101, 2002.

[4] I. V. Belykh, E. Lange, M. Hasler. Synchronization of bursting neurons: what matters in the network topology, Phys. Rev. Lett., 94: 188101, 2005.

[5] S. Boccaletti, V. Latora, Y. Moreno, M. Chavez, D. U. Hwang. Complex networks: structure and dynamics, Physics Reports, 424: 175-308, 2006.

[6] L. Donetti, P. I. Hurtado, M. A. Muñoz. Entangled networks, synchronization, and optimal network topology, Phys. Rev. Lett., 95: 188701, 2005.

[7] Z. S. Duan, G. Chen, L. Huang. Analysis and control of network synchronizability, arXiv:0706.2901v1, 2007.

[8] C. Godsil, G. Royle. Algebric Graph Theory, Springer-Verlag, New York, 2001.

[9] P. Holme, B. J. Kim. Vertex overload breakdown in evolving networks. Phys. Rev. E, 65: 066109, 2002.

[10] H. Hong, B. J. Kim, M. Y. Choi, H. Park. Factors that predict better synchronizability on complex networks, Phys. Rev. E, 69: 067105, 2004.

[11] L. Kocarev, P. Amato. Synchronization in power-law networks, Chaos, 15: 024101, 2005.

[12] W. Lu, T. Chen. Synchronization analysis of linearly coupled networks of discrete time systems, Physica D, 198: 148-168, 2004. 
[13] J. H. Lü, X. H. Yu, G. Chen, D. Z. Cheng. Characterizing the synchronizability of small-world dynamical networks, IEEE. Trans. Circuits Syst.-I , 51(4), 787-796, 2004.

[14] R. Merris. Laplacian matrices of graph: a survey, Linear Algebra and its Applications, 197-198: 143-176, 1994.

[15] R. Merris. Laplacian graph eigenvectors, Linear Algebra and its Applications, 278: 221-236, 1998.

[16] A. E. Motter, C. S. Zhou, J. Kurths. Enhancing complex-network synchronization, Europhysics Letters, 69(3): 334-340, 2005.

[17] V. Nikiforov. Eigenvalues and extremal degrees of graphs, Linear Algebra and its Applications, 419: 735-738, 2006.

[18] T. Nishikawa, A. E. Motter. Maximum peformance at minimum cost in network synchronization, Physica D, 224: 77-89, 2006.

[19] T. Nishikawa, A. E. Motter, Y. C. Lai, F. C. Hoppensteadt. Heterogeneity in oscillator netwroks: Are smaller worlds easier to synchronize? Phys. Rev. Lett., 91(1): 014101, 2003.

[20] Y. L. Pan. Sharp upper bounds for the Laplacian graph eigenvalues, Linear Algebra and its Applications, 355: 287-295, 2002.

[21] L. M. Pecora, T. L. Carroll. Master stability functions for synchronized coupled systems, Phys. Rev. Lett., 80(10): 2109-2112, 1998.

[22] F. Sorrentino, M. di Bernardo, F. Garofalo, G. Chen. Controllability of complex networks via pinning, Phys. Rev. E, 75: 046103, 2007.

[23] A. Stefański, P. Perlikowski, T. Kapitaniak. Ragged synchronizability of coupled oscillators, Phys. Rev. E, 75: 016210, 2007.

[24] X. Wang, Y. C. Lai, C. H. Lai. Synchronization is hierchical cluster networks, arXiv. nlin. $C D / 0612057 v 1,2006$.

[25] X. F. Wang, G. Chen. Synchronization in scale-free dynamical networks: robustness and fragility, IEEE Trans. Circuits Syst-I, 49(1): 54-62, 2002.

[26] D. J. Watts, S. H. Strogatz. Collective dynamics of 'small-world' networks, Nature, 393(6684): 440-442, 1998.

[27] C. W. Wu. Synchronizability of networks of chaotic systems coupled via a graph with a prescribed degree sequence, Phys. Lett. A, 346: 281-287, 2005. 
[28] M. Zhao, T. Zhou, B. H. Wang, G. Yan, H. J. Yang, W. J. Bai. Relations between average distance, heterogeneity and network synchronizability, Physica A, 371(2): 773-780, 2006.

[29] C. S. Zhou, J. Kurths. Dynamical weights and enhanced synchronization in adaptive complex networks, Phys. Rev. Lett., 96(16): 164102, 2006. 